\title{
Protocol For a Randomised Controlled Feasibility Study Examining the Efficacy of Brief Cognitive Therapy For the Treatment of Panic Disorder in Adolescents (PANDA)
}

\section{Polly Waite ( $\sim$ p.l.waite@reading.ac.uk)}

The Departments of Experimental Psychology and Psychiatry, University of Oxford

\section{Study Protocol}

Keywords: Panic disorder, adolescent, young people, youth, cognitive therapy, CBT, psychological treatment, brief

Posted Date: September 30th, 2021

DOI: https://doi.org/10.21203/rs.3.rs-934168/v1

License: (c) (1) This work is licensed under a Creative Commons Attribution 4.0 International License. Read Full License

Version of Record: A version of this preprint was published at Pilot and Feasibility Studies on March 3rd, 2022. See the published version at https://doi.org/10.1186/s40814-022-01009-z. 


\section{Abstract}

Background: Panic disorder occurs in between 1-3\% of adolescents, is associated with high levels of comorbidity, and without treatment, appears to have a chronic course. To improve access to effective psychological interventions, briefer versions of CBT have been developed and evaluated for preadolescent children with anxiety disorders. However, there are currently no brief evidence-based CBT interventions for adolescents with anxiety disorders that can be delivered in less than eight sessions. Given that a brief version of cognitive therapy has been shown to be effective in adults with panic disorder, with a large effect size, it is possible that an adapted version could be effective for adolescents with panic disorder.

Methods: The study will examine whether a definitive trial can be conducted, based on a feasibility RCT using several well-defined criteria. The feasibility RCT is a single centre, randomised control trial. Between 30-48 young people (age 11-18 years) who meet diagnostic criteria for panic disorder, attending a routine clinical service, will be randomly allocated to receive either (i) brief cognitive therapy, or (ii) a general form of CBT treatment that is more commonly used for adolescents with anxiety disorders. Both will be delivered $1: 1$ by a therapist and involve five treatment sessions and two booster sessions. Young people's outcomes will be assessed at the end of treatment and at 3 month follow up, and qualitative interviews will be conducted to examine acceptability. We will also explore outcomes 1 -year after the completion of treatment.

Discussion: This study will test the feasibility of a randomised controlled trial to compare brief cognitive therapy to a general form of CBT for adolescents with panic disorder in the UK. The outputs from the study will provide a clear indication of the feasibility of a future definitive trial and, if indicated, the critical resources that will be required and key information to inform the design and maximise the successful completion of the trial. This has the potential to bring direct benefits to young people and their families, as well as services and society more broadly.

Trial Registration: This trial is registered on the ISRCTN Registry, registration number ISRCTN14884288, registered retrospectively on 05/12/2019.

\section{Introduction}

For many people, panic disorder begins in adolescence (1), with between $1-3 \%$ of adolescents aged $11-$ 19 years meeting diagnostic criteria for panic disorder $(2,3)$. This early onset may be associated with more severe symptoms and a worse outcome than panic disorder that starts in adulthood (4). Young people with panic disorder commonly avoid a range of environments, including school cafeterias or restaurants, small rooms and auditoriums (5), which is likely to have a significant impact on functioning at school and with friends. Furthermore, panic disorder in adolescence has high rates of comorbidity, including with other anxiety disorders and depression, and is associated with the subsequent onset and 
persistence of alcohol abuse and dependence (5-7). Left untreated, it appears to have a chronic course (6). These factors highlight the need for effective and accessible treatments.

Two psychological treatments have been extensively evaluated with adults with panic disorder and shown to be similarly effective in their original format (i.e. 12 to 15 sessions): panic control treatment (PCT) (8), and cognitive therapy (CT) for panic disorder (9). Both treatments have been shown to be superior to a range of other treatments including relaxation therapy, supportive psychotherapy, and medication (10). However, when briefer forms of the two treatments were developed and evaluated, only the brief form of CT remained as effective as the full treatment $(9,11)$. Specifically a version of CT involving self-study modules and 5 sessions (plus two booster sessions) of therapy (B-CT) did not differ significantly from the full 12 session (plus two booster sessions) treatment (F-CT) immediately or at 12month follow up (panic-free at post-treatment: F-CT 79\%, B-CT 71\%; 12-month follow-up: F-CT 71\%, B-CT $79 \%$ ), with $0 \%$ dropping out of treatment and large effect sizes for both the full and brief treatments (posttreatment: F-CT $d=2.9$, B-CT $d=2.9$; 12-month follow-up: F-CT $d=2.8$, B-CT $d=3.2$ ).

In contrast to treatment for adults, the treatment of panic disorder in adolescents has been largely neglected and a considerable number of randomised controlled trials treating anxiety in children and adolescents have excluded young people who have panic disorder as the primary problem (12-14). The most substantial evidence base for CBT for a broad range of anxiety disorders in children and adolescents comes from trials using a general, transdiagnostic treatment approach (e.g., the 'Coping Cat' treatment protocol (15), or the adolescent version, the 'C.A.T. Project' (16)). The treatment involves anxiety management (e.g., psychoeducation, cognitive restructuring, and relaxation techniques) prior to graded exposure, where the young person learns to face their fears through exposure to the feared situation or stimulus in a graded way. Meta-analyses have demonstrated that around $50 \%$ of children and adolescents are free of their primary diagnosis at the end of CBT (17). However, outcomes for young people with panic disorder specifically are unknown. In the UK, CBT appears to be the most used treatment approach by clinicians in routine clinical practice to treat young people with panic disorder. However, a recent national survey of over 400 clinicians working in Child and Adolescent Mental Health Services (CAMHS) in the National Health Service (NHS) found that only $2.6 \%$ were able to identify any specific treatment protocols to treat young people with panic disorder, either within transdiagnostic or disorder-specific treatments (18).

Currently, young people face significant difficulties to access evidence-based treatments. Fewer than one in five adolescents in need of treatment receive appropriate psychological interventions (19), and many face significant delays or spend months on waiting lists for treatment within routine clinical services (20). In order to improve access to effective psychological interventions, briefer versions of CBT have been developed that can be delivered by non-specialists, so that more intensive treatments can be reserved for those who do not, or who are unlikely to, benefit from a brief treatment (21). Brief CBT has recently been defined as referring to either low intensity CBT (i.e., six hours or less of contact time with a therapist, using self-help materials) and/or brief high intensity CBT (i.e., based on the standard evidence-based CBT treatment, with therapy contact time $50 \%$ or less than the full CBT intervention) (22). Suitable brief CBT 
treatments have been developed and evaluated for pre-adolescent children (23), however there has been limited research attention on brief CBT interventions for adolescents with anxiety disorders. Of the sixteen studies of psychological therapies for adolescents with anxiety disorders (including panic disorder) identified in a recent meta-analysis (24), only one study (25), involving adolescents with a range of anxiety disorders, fulfilled the definition of brief CBT.

Many treatments for anxiety disorders in children and adolescents have successfully been adapted from treatments shown to be effective in adults. Although Barlow \& Craske's PCT has been successfully adapted for adolescents with panic disorder in the U.S. (PCT-A) (26), the two derivations of treatment require a considerable amount of therapist time (between 11 and 22 hours of therapy) $(27,28)$. In addition, the intensive form of treatment, which has the most empirical support, would be difficult to implement in NHS CAMHS settings as it requires treatment to be conducted by a therapist for several hours over consecutive days. To date, there has been no adaptation of Clark's cognitive therapy for adolescents with panic disorder, despite emerging evidence that the psychological processes targeted in CT for adults may also be evident in adolescents (29-31). Given that the brief version of this treatment has been shown to be effective in adults with panic disorder, with a large effect size, it is possible that recovery rates for adolescents could be substantially improved, and treatment considerably shortened by using this alternative treatment approach, adapted for use with adolescents.

To maximise the likelihood of a future successful large scale randomised controlled trial (RCT), the aim of this study is to test the feasibility of an RCT to compare brief cognitive therapy for panic disorder (adapted for adolescents) to a general form of CBT treatment that is more commonly used for adolescents with anxiety disorders, including panic disorder, in the UK.

\section{Method}

\section{Aim}

The specific aims of this study are to:

a. Identify appropriate clinical outcome and economic measures for a subsequent definitive trial;

b. Explore the acceptability of the treatments and trial procedures;

c. Establish likely recruitment rates;

d. Establish the likely rate of treatment drop out;

e. Establish likely retention to research assessments post treatment and at 3-month follow-up;

f. Explore retention to a brief 12-month follow-up;

g. Establish if brief cognitive therapy can be delivered so that it is clearly distinct from a general form of $\mathrm{CBT}$, with high levels of fidelity by practitioners and credibility with patients in both arms; 
h. Conduct exploratory analyses of possible outcomes for the two treatments including changes in anxiety symptoms, diagnostic status, quality of life, healthcare resource use and other outcomes identified through Patient and Public Involvement (PPI);

i. Describe negative impacts of the treatments and the trial procedures (to patients, their parents and clinicians);

j. Explore young people's outcomes on measures of symptom and functional impairment.

\section{Design}

This study is a parallel design RCT comparing brief cognitive therapy to a general form of CBT treatment that is more commonly used for adolescents with anxiety disorders. It will be conducted within the Anxiety and Depression in Young people (AnDY) Research Clinic at the University of Reading. Young people and their parent/carer's expectations of treatment will be assessed prior to the beginning of treatment using a brief questionnaire (32). Additionally, qualitative interviews will be conducted with a subsample of participating young people post-treatment to explore their experience of treatment. We will also interview parents/carers and key stakeholders (e.g., service managers, study clinicians and referrers) about their experiences.

Young people, including service users (with panic disorder and/or other anxiety disorder), parents and stakeholders have been involved in all stages of the study. Young people have been involved in adapting the questionnaires and workbooks used to support cognitive therapy and advising on recruitment.

Parents have been consulted on the development of an accompanying workbooks for parents/carers for those in the cognitive therapy arm. Stakeholders, including teachers and GPs, have advised on recruitment and screening procedures.

\section{Feasibility criteria}

The outputs from the study will provide a clear indication of the feasibility of a future definitive trial and, if indicated, the critical resources that will be required and key information to inform the design and maximise the successful completion of the trial. To feel confident that a definitive trial can be delivered we would require the following criteria to be met:

a. Serious negative impacts (e.g., worsening of symptoms, significant increase in risk as determined by clinical judgment of the treating clinician) do not occur as a result of participation in the trial;

b. There are no serious concerns about the acceptability of the trial procedures;

c. A generalisable sample can be recruited which will maintain study equipoise (i.e., at least $80 \%$ of eligible participants will agree to randomisation);

d. Treatment drop-out rates will be no more than $20 \%$; 
e. At least $80 \%$ of participants will complete all assessments, including the 3 -month follow-up (to maximise generalizability for a larger trial);

f. Treatment delivered within the brief cognitive therapy and general CBT treatment arms will be clearly distinct in a manner that indicates therapist adherence to the manuals (with sessions containing at least $80 \%$ 'allowable' and less than $20 \%$ 'not-allowable' features of the prescribed treatment).

\section{Setting}

Participants will be recruited to the study through the Anxiety and Depression in Young People (AnDY) Research Clinic at the University of Reading, a clinical service that is funded by local NHS commissioning. The AnDY Research Clinic offers assessments, treatment and research to children and young people who are experiencing difficulties with anxiety and/or depression. Assessment and treatment sessions will be conducted either face to face within the clinic or via video-conferencing software, whenever restrictions due to the coronavirus pandemic prohibit face to face meetings.

\section{Participants}

Between 30 and 48 participants will be recruited to the feasibility study. To be included in the study, the young person must be aged 11-18 years at assessment and meet diagnostic criteria for panic disorder (33), as either the primary or secondary presenting disorder. They must have had at least one panic attack in the month prior to assessment. If the young person has a co-morbid medical condition (such as asthma, epilepsy, or cardiovascular disease), the young person's GP must have been consulted and given the opinion that this will not interfere with treatment delivery. They must not be taking psychotropic medication or alternatively, they must be willing to be withdrawn from medication before the start of the trial under the supervision of their GP. A minimum 6-week drug-free period for SSRIs and a 2-day drug-free period for benzodiazepines will be required before the young person can start treatment and they must agree not to start medication during the trial. They must be able to speak English, willing to accept random allocation and engage in the treatment. Young people will be excluded if they have a co-morbid condition that is likely to interfere with treatment delivery, such as an established autistic spectrum disorder, learning disabilities, suicidal intent, or recurrent or potentially life-limiting self-harm (i.e., current frequency of at least once per week or self-harm that requires medical attention), they been identified by social services as currently 'at risk' due to, for example, child protection concerns or if they are receiving a psychological intervention, or have received previous treatment with cognitive therapy or CBT for panic disorder.

\section{Sample size}

As is typical in feasibility studies, the sample size is not based upon a power calculation (34). Across both groups, we will aim to recruit 48 participants, but will accept a minimum of 30 participants. If adverse events or significant deterioration were likely to occur as a result of participating in the trial this would be expected to be observable within this sample size and there will be sufficient throughput of 
potential participants within the recruitment period to examine recruitment and retention rates and participant flow through the trial, and to examine treatment integrity (35-37). The proposed sample size is also sufficient to provide an estimate of the variation in outcomes on which to power a definitive trial, if indicated, based on continuous outcomes (i.e., panic disorder severity symptoms).

A sub-sample will be involved in qualitative interviews after the treatment has been delivered. We will use purposive sampling and sample for a diverse range of demographic and clinical characteristics. The adequacy of the sample size will be continuously evaluated during the study and recruitment to the interviews will come to an end when the sample holds sufficient information power to develop new knowledge $(38,39)$. However, it may also be shaped and constrained by the number of potential interviewees, particularly in the stakeholder sample, as well as time and resources available (39). At this stage, it is anticipated that this is likely to involve around $10-15$ young people, $10-15$ parents, as well as 5-10 stakeholders.

\section{Procedure}

The study procedure is in line with the Standard Protocol Items: Recommendations for Interventional Trials (SPIRIT) statement 2013 (40). Figure 1 shows the schedule of enrolment, interventions, and assessments according to the SPIRIT statement, and the SPIRIT checklist can be found in the electronic supplementary materials. Figure 2 provides an overview of the study procedures.

\section{Recruitment}

We will recruit eligible participants who have been referred to the clinic where the study is taking place. In addition, we will contact local primary/secondary care services, mental health services for children and young people, and schools to advertise the trial and request referrals. We will also advertise the trial through social media, local newspapers, and radio. Potential participants identified through these outreach activities will be asked to complete a screening form and indicate whether they would like treatment. If their responses indicate they may be eligible, the young person and their parent/carer will receive a telephone triage by the clinical team. If the triage indicates that they are likely to meet the inclusion criteria for the study, the young person will then be referred to the clinic. As is routine in the clinic, all potential participants will be invited to complete a diagnostic assessment to determine whether they meet diagnostic criteria for panic disorder and/or other anxiety disorders or depression. Where possible, the young person's parent/carer will also undertake a diagnostic assessment reporting on their child's difficulties. The young person and their parent/carer will be assessed separately. Assessments will be carried out by members of the team who are trained to reliability and will receive supervision for every assessment from a senior assessor with extensive experience of delivering and supervising diagnostic assessments and proven reliability. Assessments will be carried out by telephone or video call if face to face appointments are not possible. Adolescents and their parent/carer will also be asked to independently complete self-report questionnaires, reporting on the adolescent's symptoms. 
If the young person is eligible for the trial, they will be sent information leaflets (adolescent and parent/carer versions) and will then meet with a member of the research team to discuss the study further. If they agree to participate, written informed consent will then be given by the parent and the young person (or assent for young people under 16 years of age). Screening logs will be maintained for eligible participants not recruited, to inform acceptability of the study to young people. Reasons for nonparticipation in the trial will be collected anonymously.

\section{Randomisation}

Consenting participants will be randomised to receive individual sessions of brief cognitive therapy or standard CBT. To protect against bias, randomisation will be performed using an online randomisation system. Participants will be randomised to receive either cognitive therapy or standard CBT, with a randomisation ratio of 1:1 and stratification based on the key baseline feature that is likely to be associated with outcome (i.e., panic disorder symptom severity). Participants will be informed of their allocation immediately following their consent to take part in the study.

\section{Treatment}

Once randomised, participants will be allocated to a clinician for the relevant treatment arm. Clinicians delivering the trial interventions will be qualified therapists (e.g., Child Psychological Wellbeing Practitioners, Psychological Wellbeing Practitioners or Clinical Psychologists) and will only deliver treatment in one arm of the trial to prevent contamination. In line with good clinical practice (41), the young person will complete self-report measures prior to each treatment session to inform treatment.

\section{Post-treatment and follow up}

As well as completing measures pre-treatment (and for young people, prior to each session), young people and their parents/carers will complete measures at the end of the main treatment sessions (posttreatment) and at 3-month follow up. A further measure will be completed by young people 1-year following the end of treatment. The post treatment and follow up measures will be completed in the clinic, or if this is not possible, may be taken home and emailed or posted back to the clinic, or completed online. At the 3-month follow-up assessment, participants will also have a further diagnostic assessment with an assessor who will not know which treatment they received. For participants who have discontinued with the treatment they were allocated to at randomisation, this follow up assessment will be conducted at the time when it should have occurred had they continued in that treatment arm. Participants will all be reimbursed with a voucher for their time and inconvenience in completing additional measures at pre- and post-treatment, and follow-up assessments.

A sub-sample of young people and parents/carers will be invited to take part in a qualitative interview to discuss their experiences of treatment and the research study. We also aim to recruit key stakeholders involved in the research study. This will include service managers, clinicians involved in both treatment arms, referrers from a range of settings (e.g., schools and primary care) and professional backgrounds. 
Interviews will be conducted with participants individually and will be conducted by psychology students who have had training in qualitative research and will receive supervision from researchers with expertise in this approach. Interviews will take place either face to face, by video call or telephone call. A purposive sampling strategy will be adopted to identify participants who differ on demographic variables. For example, for the young people, this will include age, severity of panic disorder, level of impairment and presence of comorbid difficulties at initial assessment, treatment outcomes and number of sessions attended. Participants who take part in the interviews will be reimbursed for their time and inconvenience in attending the interviews.

All data will be stored separately from other personally identifying data and will not be shared outside the research team to ensure participant confidentiality. Identifiable participant personal information will be stored on a secure, restricted access drive. All other data will be identified using a number, and the file with information linking numbers and names will be stored separately in a password-protected file on the University's server.

\section{Intervention}

\section{Brief cognitive therapy}

This treatment was developed for the treatment of panic disorder by Professor David Clark and colleagues (42). A variety of procedures described in a manual (43) will be used to reverse the maintaining factors identified in Clark's (1985) cognitive model of panic disorder. These procedures will be introduced in four self-study modules. Prior to each of the first four sessions, the young person will read a self-study module and complete the written exercises and homework activities outlined in the module. These modules have been adapted to be suitable for adolescents, with PPI input, e.g., changing the case studies from adults to young people, changes to the language and metaphors to be developmentally appropriate, and adding graphics to make them visually appealing. Additional handouts dealing with common catastrophic thought (e.g., 'I'll faint' or 'I'll lose control') may also be used if relevant to the young person's concerns. In the session, the therapist will discuss the module with the young person and clarify any misunderstandings. The focus of the session will then be on experiential exercises in which bodily sensations and safety behaviours are systematically manipulated to demonstrate their adverse effects, as well as behavioural experiments in which the young person tests pre-specified negative predictions while dropping their safety behaviours. Process measures will be used in each session to generate meaningful behavioural experiments. A habituation rationale will not be used, repeated exposure to the same situations not encouraged, and the person will not be encouraged to develop and use positive self-talk before or during behavioural experiments. The treatment will involve five sessions of 30-90 minutes, with up to two 60-minute booster sessions over the following three months.

Parents/carers will be encouraged to read the self-study modules to learn about panic disorder and cognitive therapy techniques and asked to support their child in completing the self-study module each 
week and carrying out behavioural experiments (or other relevant activities) between sessions. They will also be given a parent/carer self-study module, with advice on how they can support their child during the treatment. At the end of sessions, they will typically be involved in the relapse prevention plan, so that they can support their child with this after treatment ends. The therapist will also liaise with school at assessment and throughout therapy where necessary to support the young person in treatment. This may include providing the school with psychoeducation about panic disorder, cognitive therapy techniques, and how school staff can support at different stages of the treatment (e.g., planning school-based behavioural experiments).

\section{General CBT}

Treatment will involve anxiety management techniques (e.g., psychoeducation about anxiety, breathing retraining and relaxation), before moving on to the development of an exposure hierarchy, in which the young person will develop, with their therapist, an ordered list of feared stimuli according to their anticipated fear reaction. They will then begin to face their fears by putting themselves in situations that they worry may trigger panic attacks, beginning with the least anxiety-proving situation, and learning that over time their anxiety diminishes. Once they can experience the situation without experiencing significant anxiety they will then move on to the next situation on the list. Graded exposure is based on the idea that with repeated exposure to feared stimuli, the person will habituate and experience a reduction in fear. This leads them to learn a new set of associations and learn that they can cope. As is typical in routine clinical practice, clinicians will use worksheets that are freely available on the Internet to support the treatment. The scheduling and length of treatment sessions will be the same as the brief cognitive therapy treatment.

Parents/carers will be given psychoeducation about anxiety management and graded exposure. They will be asked to support their child outside session in carrying out exposure tasks, and with the relapse prevention plan at the end of treatment. The therapist will also liaise with school at assessment and throughout therapy as necessary to support the young person in treatment.

\section{Measures and assessment Screening questions}

The screening questions can be found in the electronic supplementary materials. If potential participants respond yes to all items on the screening form, and indicate that they would like help, they will be invited to a telephone triage. Some discretion will be allowed, and therefore young people who have responded 'no' to some items may still be invited to a telephone triage with a clinician to gain further information. If panic disorder is indicated, they will then receive a diagnostic assessment.

\section{Demographic information}

Demographic information will be collected from the parent on the pre-treatment questionnaire, and this will include information about the young person (age, gender, ethnicity, developmental history, past/current medical conditions, past/current treatment for psychological difficulties) and the parent 
(relationship to young person, age, relationship status, education and employment). This will be used to describe the sample.

\section{Diagnoses of anxiety disorders and comorbid disorders}

A diagnostic assessment will be used to establish if the young person reaches diagnostic criteria for any anxiety disorders (including panic disorder) and mood disorders, and to determine the primary presenting problem. A structured diagnostic interview will be carried out at baseline and 3-month follow up. The anxiety section of the Anxiety Disorders Interview Schedule (ADIS-C/P; 44) will be used to determine whether the young person meets diagnostic criteria for any anxiety disorders, including panic disorder, and/or behavioural disorders, and to establish a clinician rating of severity for each disorder (CSR). The pre-treatment diagnosis with the highest CSR will be classed as the primary diagnosis. All final diagnoses and CSRs will be determined by consensus with a supervisor with proven reliability. Additionally, mood disorders will be assessed using the relevant sections of the Kiddie Schedule for Affective Disorders and Schizophrenia (K-SADS; 45), which is a structured diagnostic interview for DSM-IV affective disorders and schizophrenia.

\section{Symptoms of panic, anxiety and depression}

The Panic Disorder Severity Scale for Children and Adolescents (PDSS-A; 46) will be administered to assess change in the frequency and severity of adolescents' panic disorder symptoms and anticipatory anxiety and associated agoraphobia, avoidance, fear, work and social impairments. There are seven items; each rated on a 0-4 scale, with a higher score indicated greater severity. It has been shown to have good psychometric properties with an adolescent population (46).

The Revised Child Anxiety and Depression Scale (RCADS; 47) will be used to measure symptoms of anxiety disorders and depression. This will be completed by the adolescent at every treatment session and by the adolescent and their parents/carers at pre, post, and follow up appointments. The RCADS is a 47-item parent and child report scale which assesses symptoms of separation anxiety disorder, social anxiety disorder, generalized anxiety disorder, panic disorder, obsessive compulsive disorder and major depressive disorder. Responders rate how often each item applies on a scale of 0 ('never') to 3 ('always'). The RCADS has been shown to have robust psychometric properties in children and young people from 7-18 years of age (48).

\section{Functional Impairment}

The Child Anxiety Impact Scale (CAIS; 49) will be used to determine the extent to which anxiety interferes in the young person's life. This will be completed at pre, post and follow up appointments by young people and parents/carers. This measure covers three psychosocial domains (academic, social activities and home/family environments) and consists of 27 items rated on a 4-point scale There are versions for children/adolescents and parents to complete, both of which have been shown to have good psychometric properties $(49,50)$. Internal consistency for the CAIS-C/P was good to excellent across assessment time points (CAIS-C $a=.85-.99$; CAIS-P $a=.93-.95)$. 
The Clinical Global Impression Scale - Improvement (CGI-I; 51) will be used after the 3 month follow up assessment to assess the young person's post-treatment changes in global functioning. This requires the assessor to rate how improved the patient is compared to their initial assessment, prior to treatment, on a scale of 1 (very much improved) to 7 (very much worse). Final scores will be dichotomised to represent 'much or very much improved' versus other. A second rater will independently rate the CGI-I for all participants to establish inter-rater reliability.

\section{Panic disorder specific measures}

Participants will complete panic disorder-specific measures to assess symptoms, cognitions, and safety behaviours pre-treatment, post-treatment, and at 3-month follow-up. The measures that have been designed for use with adults have been adapted for use with adolescents based on consultation with service users. In the cognitive therapy treatment arm, participants will also complete the measures prior to each session, to guide the treatment sessions.

The Body Sensations Questionnaire (52) is a self-report measure of bodily symptoms relating to panic that an individual may experience and the level of fear they cause. This will be used to determine the degree to which participants are experiencing panic sensations. Respondents are asked to rate the level of fear they feel when experiencing 18 different bodily sensations, on a five-point scale.

The Agoraphobia Cognitions Questionnaire (52), modified by Clark and colleagues (42) will be administered to measure cognitions associated with panic disorder. This measure includes 18 cognitions commonly associated with panic disorder. First, the respondent rates the frequency with which the thought occurred on a scale of 1 ('thought never occurs') to 5 ('thought always occurs when I am anxious'). Second, the respondent rates the extent to which the thought was considered to be true on a scale of 0 ('I do not believe this thought') to 100 ('I am completely convinced this thought is true'). The ACQ has high construct and discriminant validity in adults (52) but to date, there is no psychometric data on the use of this measure with adolescents.

The Safety Behaviours Questionnaire (43) will be administered to identify panic related safety-seeking behaviours. Adolescents will be asked to complete the self-report version. This involves 16 items, each rated on a 4-point scale from 'never' to 'always'.

The Mobility Inventory (53) is a validated measure of avoidance behaviour and associated panic attacks. Adolescents will be asked to complete the self-report version. Respondents are asked to rate how often they avoid situations both when they are on their own and when they are other people on a five-point rating scale (never/rarely/half of the time/most of the time/always).

The Panic Diary (43) will be given to adolescents in the cognitive therapy arm to fill in on a daily basis as part of treatment to record the frequency of panic attacks, identify triggers, sensations and thoughts, and to help them to answer their panic related thoughts during an attack. At the beginning of therapy, patients only complete the left-hand side of the diary. As proficiency in answering panic related thoughts develops, the whole diary is completed. 


\section{Session by session measures to guide treatment}

The Outcome Rating Scale (ORS; 54) will be used to assess functioning across different areas of the young person's life. It has four items: symptom distress, interpersonal wellbeing, social role, and overall wellbeing. Each item is rated using a 10-centre visual analogue scale, with instructions to place a mark on each line. A higher score indicates better functioning. It has good reliability and validity with an adolescent population (55).

The Session Rating Scale (SRS; 56, 57) assesses key dimensions of an effective therapeutic relationship and is given at the end of each therapy session to get feedback from young people and parents/carers so that any issues related to therapeutic alliances can be immediately identified and addressed. It comprises four rating scales (relationship with the therapist, goals and topics, approach or method and an overall rating) and uses the same visual analogue scales as the ORS. It has well-established reliability and validity $(57,58)$.

The Goal Based Outcomes tool (59) enables the young person to set up to three goals at the beginning of treatment as a way of evaluating their progress on a Goal Progress Chart. Progress towards individual goals is then periodically rated on a scale from 0 (no progress) to 10 (goal has been reached). Although this measure is now widely used in CAMHS, its psychometric properties have not yet been established.

\section{Satisfaction with care}

At the 3-month follow up assessment, participants will rate their satisfaction with the service they have received using the Experience of Service Questionnaire (ESQ; 60), a measure that was developed by the Health Care Commission as a means of measuring satisfaction in Child and Adolescent Mental Health Services. There are versions for young people and their parents/carers to report on the extent to which they agree with 12 statements examining what the respondent liked about their care and the environment, what they felt needed improving, and three free text sections for any other comments. It is routinely used within child and adolescent mental health services and has been demonstrated to have good psychometric properties (61).

\section{Health economic measures}

Health economic self-report questionnaires as detailed below are collected from parents/carers and young people at the pre, post and 3-month follow up. Clinicians will use logs at each treatment and supervision session and any other times as required.

A societal perspective for costs will be adopted and patient level resource use data will be collected from parents/carers on a Client Services Receipt Inventory (CSRI) using patient-health diaries to facilitate recall of healthcare resource use and from clinicians' logs. This data will be provided by clinicians and parents/carers and will include all health and social care cost-generating resources (e.g., staff time for provision of treatment, training and supervision, GP use, referrals, and other relevant services identified), 
non-NHS cost-generating services (e.g. educational services) as well as leisure and lost productivity time estimates for the parents/carers (e.g. days off school/college/work).

The EQ-5D (5L) (62) is a well-validated preference-based measure of health-related quality of life, designed to estimate quality adjusted life years (QALYS), that is widely used across disease areas. The EQ-5D questionnaire contains five simple questions each concerned with a different domain of everyday life, i.e., mobility, self-care, usual activities, pain/discomfort, and anxiety/depression. For each domain the respondent must indicate whether he/she experiences no problems, slight problems, moderate problems, severe problems, or extreme problems. The respondent's answers provide a description or profile of the respondent's quality of life, and a weight or value can then be placed on each profile using an existing UK tariff derived from the general public $(62,63)$. The full questionnaire also includes a visual analogue scale (VAS) for participants to rate their overall health on a scale from 0 ("worst imaginable health") to 100 ("best imaginable health"). Quality of life of carers will be assessed using the EQ-5D-5L self-report. The EQ-5D-Y $(64,65)$ was adapted directly from the EQ-5D to estimate utility values for young people (from 8 years). It covers the same domains as the EQ-5D, but the wording of the questions in each dimension is modified to make it appropriate to a younger age range. The EQ-5D (-Y; $-5 \mathrm{~L})$ has established feasibility and reliability $(62,63)$.

The Child Health Utility 9D (CHU-9D; 66, 67) is a paediatric measure of health-related quality of life, which allows the calculation of QALYs for use in cost utility analysis. It includes nine dimensions (worried, sad, pain, tired, annoyed, schoolwork, sleep, daily routine, activities) each with five levels. The measure was originally developed with children aged 7-11 years, and subsequently validated in an adolescent population $(11-17$ years) $(67,68)$. The CHU-9D is also available in a 'proxy' version for parent/ carer completion, and this will also be used.

\section{Treatment credibility}

Participant expectancies and views regarding treatment credibility will also be assessed prior to treatment through a credibility and expectation of improvement scale (32). This consists of three items, rated on a scale from 0 (not at all) to 10 (completely), asking about how logical the treatment seems, confidence in its success at reducing their symptoms, and their likelihood to recommend the therapy to a friend with similar symptoms.

\section{Distinctness of therapies}

To establish that the therapies in each arm are distinct from one another, a checklist of the components of each therapy will be given to therapists to complete at the end of every treatment session. The checklist has been designed for this trial and includes items that are distinct to either brief cognitive therapy (e.g., behavioural experiments designed to test out beliefs) or general CBT (e.g., development of a fear hierarchy). Therapists will indicate which components were carried out in the session that they have just completed. The ratings will be used to compare the content of the treatment sessions in the two arms to determine their distinctiveness. 


\section{Qualitative interviews}

Semi-structured qualitative interviews will be conducted with the young people post-treatment with both groups to explore their experiences of treatment and the research trial, as well as parents/carers and stakeholders. Interviews will be one to one, conducted face to face, by video call or by phone, and will be recorded using digital audio recording (e.g., MP3) or through Microsoft Teams and then transcribed. Interviews will follow predetermined topic guides (see electronic supplementary materials). During interviews with young people about treatment, materials such as the participant's therapy folder and flashcards of different core components of treatment may be used to assist the young person in talking about their experience and expressing their views about elements of treatment.

\section{Data analysis}

Analyses will primarily investigate recruitment and retention rates presented as a CONSORT diagram providing both overall and individual arm results at all assessment points. Clinical outcomes will be represented using descriptive statistics for each study arm. An exploratory comparison of between group differences on the clinical outcome measures will be undertaken to assess whether the observed effect size is in line with our expected effect based on the literature, using analysis of covariance or a suitable alternative. Ninety-five per cent confidence intervals will be constructed for the between group differences for each of the outcomes, adjusted for baseline, and compared with the literature. The number and percent of missing data per outcome, group and time point will be summarised. No imputation methods will be used. Where available, the overall baseline clinical data will be compared with routinely available service level data for adolescents with a primary diagnosis of an anxiety disorder to assess the representativeness of trial participants. The results of the feasibility trial will also be used to develop, refine, and test a full statistical analysis plan for use in the subsequent main trial.

Suitability and acceptability of the economic measures will be assessed based on both rates of responses at the end of the feasibility study and from young peoples' and their parents/carers' feedback. Proportions of responses to healthcare resource use and health outcome measure questions will be presented in separate tables for the two treatment arms. Missing data will be explored to establish whether this is due to lack of response to specific questions, to the measure altogether, or to loss of follow up. Rates of this missing data will also be compared to that of clinical measures to assess patterns in the response of certain participants. For both quality-of-life measures (i.e., the EQ-5D-Y and the CHU-9D), utility scores and quality adjusted life years (QALYs) will be calculated and compared for both treatment groups to explore how sensitive each measure is to change over time. Adolescent self-report and parent/carer-report on the young person will also be compared for the CHU-9D to assess any discrepancies in responses of the adolescents and their parents. Finally, variation in quality of life as derived from the EQ-5D-5L will be reported and compared across both treatment groups.

Thematic analysis (69) will be used to identify and describe emergent themes within the qualitative interviews about the experience of receiving treatment and taking part in the study. This technique was chosen due to its flexible nature, and because it is not associated with a particular theoretical framework 
(70) and is an appropriate method for applied health research (71). Thematic analysis has been used to explore people's experiences of psychotherapy (e.g. 72,73$)$ and is suitable for analysis of this data. A number of strategies will be employed to enhance the credibility and methodological rigour of the analysis (74), such as co-analysis of transcripts and use of reflexive practices in supervisory discussion.

\section{Trial and data monitoring}

As this is a feasibility study being conducted at a single, secure site, the study investigators will be responsible for monitoring the conduct of the research, including data monitoring, managing adverse events, and any decisions relating to early termination of the trial. Additionally, the trial management team, who will hold regular review meetings, will manage the safety and efficacy of the data. The trial will be overseen by a Trial Steering Committee, which will meet before the trial begins and then at key time points during the trial.

\section{Reporting of adverse events}

All adverse events (between the time of consent to the study and the point at which the participant completes and submits the final set of follow up questionnaires) will be recorded and reported. Follow up of any adverse events by the research team will take place up until the point that appropriate procedures are completed.

\section{Discussion}

The aim of the current study is to evaluate the feasibility of an RCT to compare brief cognitive therapy to a general form of CBT for adolescents with panic disorder in the UK. This will be delivered largely by therapists, such as psychological wellbeing practitioners, trained to deliver brief evidence-based treatments and therefore able to provide cost-effective treatments within clinical services. The outputs from the study will provide a clear indication of the feasibility of a future definitive trial and, if indicated, the critical resources that will be required and key information to inform the design and maximise the successful completion of the trial. This has the potential to bring direct benefits to young people and their families, as well as services and society more broadly.

\section{Trial Status}

The study is currently ongoing. Recruitment of participants started in October 2019 and will continue until the target sample size is recruited. This is expected to be October 2021.

\section{Abbreviations}

ADIS-C/P: Anxiety Disorders Interview Schedule for Children and Parents; ACQ-adapted: Agoraphobia Cognitions Questionnaire (adapted), BSQ-adapted: Body Sensations Questionnaire (adapted), CAIS-C/P: Child Anxiety Impact Scale - child and parent version; CAMHS: Child and Adolescent Mental Health 
Service; CBT: Cognitive behaviour therapy; CGI-I: Clinical Global Impression- Improvement; CHU-9D: Child Health Utility (Paediatric Quality of Life); CONSORT: Consolidated Standards of Reporting Trials; CSRI: Client Services Receipt Inventory; DSM-5: Diagnostic and Statistical Manual of Mental Disorders, 5th edition; EQ-5D-Y: EuroQol (Quality of Life); ESQ: Experience of Service Questionnaire; KSADS-C/P: Kiddie Schedule for Affective Disorders and Schizophrenia - child and parent version; MI-adapted: Mobility Inventory (adapted); NHS: National Health Service; ORS: Outcome Rating Scale; PDSS: Panic Disorder Severity Scale for Adolescents; RCADS-C/P: Revised Child Anxiety and Depression Scale- child and parent versions; RCT: Randomised controlled trial; SBQ-adapted: Safety Behaviour Questionnaire (adapted), SMFQ: Short Mood and Feelings Questionnaire; SPIRIT: Standard Protocol Items: Recommendations for Interventional Trials; SRS: Session Rating Scale.

\section{Declarations}

\section{Ethical Approval and consent to participate}

This trial was given a favourable ethical opinion for conduct from the NHS Health Research Authority (South Central -Berkshire B Research Ethics Committee), REC reference 19/SC/0287. The University of Reading Research Ethics Committee also approved this study. The current version of the Protocol under approval is V8.0 (18/05/2021). Modifications to the trial protocol will be communicated to both these committees and updated on the trial registry. Participation in the trial is contingent on informed consent (or assent for young people under age 16) from both the young person and their parent/carer.

\section{Consent for publication}

Not applicable.

\section{Availability of data and material}

Anonymised datasets will be deposited in publicly available repositories (if available and appropriate) one year after the completion of the study.

\section{Competing interests}

The author declares that they have no competing interests.

\section{Funding}

The feasibility trial is funded by an NIHR Postdoctoral Fellowship, awarded to Polly Waite (PDF-2016-09092). The study is sponsored by the University of Reading (Dr Mike Proven, m.j.proven@reading.ac.uk). 
The funder and sponsor have had no role in the study design and will not have a role in the collection, management, analysis and interpretation of the data, writing of the final report or the decision to submit the report for publication.

\section{Authors' contributions}

Not applicable

\section{Acknowledgements}

The author gratefully acknowledges the guidance and support of Professor David M. Clark in the delivery of brief cognitive therapy, and in adapting the self-study modules and materials for use with adolescents. The author would like to thank the Trial Steering Committee (Professor Tamsin Ford, Professor Cathy Creswell, Rosie Hill, Professor Richard Meiser-Stedman and Hiroko Plant), trial managers, Dr Susie Jennings and Dr Rachel Sutton, and the AnDY Research Clinic (Dr Ray Percy, clinicians and clinic staff) for their support. Finally, the author would like to thank all the young people, parents, and stakeholders who have provided advice on various aspects of the study. The author is funded by an NIHR Postdoctoral Research Fellowship (PDF-2016-09-092). The views expressed in this publication are those of the author and not necessarily those of the NIHR or the Department of Health and Social Care. The study is sponsored by the University of Reading.

\section{References}

1. Von Korff MR, Eaton WW, Keyl PM. The epidemiology of panic attacks and panic disorder: Results of three community surveys. Am J Epidemiol. 1985;122(6):970-81.

2. Sadler K, Vizard T, Ford T, Marchesell F, Pearce N, Mandalia D, et al. Mental health of children and young people in England, 2017: Trends and characteristics. Leeds, UK; 2018.

3. Bittner A, Egger HL, Erkanli A, Costello JE, Foley DL, Angold A. What do childhood anxiety disorders predict? J Child Psychol Psychiatry. 2007;48(12):1174-83.

4. Ramsawh HJ, Weisberg RB, Dyck I, Stout R, Keller MB. Age of onset, clinical characteristics, and 15year course of anxiety disorders in a prospective, longitudinal, observational study. J Affect Disord. 2011;132(1-2):260-4.

5. Kearney CA, Albano AM, Eisen AR, Allan WD, Barlow DH. The phenomenology of panic disorder in youngsters: An empirical study of a clinical sample. J Anxiety Disord. 1997;11(1):49-62.

6. Biederman J, Faraone SV, Marrs A, Moore P, Garcia J, Ablon S, et al. Panic disorder and agoraphobia in consecutively referred children and adolescents. Journal of the American Academy of Child Adolescent Psychiatry. 1997;36(2):214-23.

7. Zimmermann P, Wittchen H-U, Höfler M, Pfister H, Kessler RC, Lieb R. Primary anxiety disorders and the development of subsequent alcohol use disorders: a 4-year community study of adolescents and 
young adults. Psychological medicine. 2003;33(7):1211-22.

8. Barlow DH, Craske MG, Meadows EA. Mastery of your anxiety and panic. New York: Graywind Publications; 1994.

9. Clark DM, Salkovskis PM, Hackmann A, Wells A, Ludgate J, Gelder MG. Brief cognitive therapy for panic disorder: a randomized controlled trial. J Consult Clin Psychol. 1999;67(4):583.

10. Gould RA, Ott MW, Pollack MH. A meta-analysis of treatment outcome for panic disorder. Clin Psychol Rev. 1995;15(8):819-44.

11. Craske MG, Maidenberg E, Bystritsky A. Brief cognitive-behavioral versus nondirective therapy for panic disorder. J Behav Ther Exp Psychiatry. 1995;26(2):113-20.

12. Hudson JL, Rapee RM, Deveney C, Schniering CA, Lyneham HJ, Bovopoulos N. Cognitive-behavioral treatment versus an active control for children and adolescents with anxiety disorders: a randomized trial. Journal of the American Academy of Child Adolescent Psychiatry. 2009;48(5):533-44.

13. Walkup J, Albano A, Piacentini J, Birmaher B, Compton S, Sherill J, et al. Cognitive behavioural therapy, Sertraline, or a combination in childhood anxiety. N Engl J Med. 2009;359:2753-66.

14. Spence SH, Donovan CL, March S, Gamble A, Anderson RE, Prosser S, et al. A randomized controlled trial of online versus clinic-based CBT for adolescent anxiety. J Consult Clin Psychol. 2011;79(5):629-42.

15. Kendall PC, Hedtke KA. The coping cat workbook: Workbook Pub.; 2006.

16. Kendall PC. The C.A.T. project manual: For the cognitive-behavioral treatment of anxious adolescents. Workbook; 2002.

17. James AC, Reardon T, Soler A, James G, Creswell C. Cognitive behavioural therapy for anxiety disorders in children and adolescents. Cochrane Database of Systematic Reviews. 2020(11).

18. Baker HJ, Waite P. The identification and psychological treatment of panic disorder in adolescents: a survey of CAMHS clinicians. Child Adolesc Mental Health. 2020;25(3):135-42.

19. Merikangas KR, He J-P, Burstein M, Swendsen J, Avenevoli S, Case B, et al. Service Utilization for Lifetime Mental Disorders in U.S. Adolescents: Results of the National Comorbidity SurveyAdolescent Supplement (NCS-A). Journal of the American Academy of Child Adolescent Psychiatry. 2011;50(1):32-45.

20. Frith E. CentreForum Commission on Children and Young People's Mental Health: state of the Nation. London: CentreForum Commission; 2016.

21. Bower P, Gilbody S. Stepped care in psychological therapies: Access, effectiveness and efficiency. Narrative literature review. Br J Psychiatry. 2005;186(1):11-7.

22. Shafran R, Myles-Hooton P, Bennett S, Öst L-G. The concept and definition of 'low intensity' cognitive behaviour therapy. Behaviour Research and Therapy. 2021:103803.

23. Creswell C, Violato M, Fairbanks H, White E, Parkinson M, Abitabile G, et al. Clinical outcomes and cost-effectiveness of brief guided parent-delivered cognitive behavioural therapy and solution- 
focused brief therapy for treatment of childhood anxiety disorders: a randomised controlled trial. The Lancet Psychiatry. 2017;4(7):529-39.

24. Baker HJ, Lawrence P, Karalus J, Creswell C, Waite P. The effectiveness of psychological therapies for anxiety disorders in adolescents: A meta-analysis. Clinical Child and Family Psychology Review. in press.

25. Stjerneklar S, Hougaard E, McLellan LF, Thastum M. A randomized controlled trial examining the efficacy of an internet-based cognitive behavioral therapy program for adolescents with anxiety disorders. PloS one. 2019;14(9):e0222485.

26. Pincus DB, Ehrenreich JT, Mattis SG. Mastery of anxiety and panic for adolescents: Riding the wave (therapist guide). Oxford: Oxford University Press; 2008.

27. Chase RM, Whitton SW, Pincus DB.. Child. Treatment of Adolescent Panic Disorder: A Nonrandomized Comparison of Intensive Versus Weekly CBT. \& Family Behavior Therapy. 2012;34(4):305-23.

28. Pincus DB, May JE, Whitton SW, Mattis SG, Barlow DH. Cognitive-behavioral treatment of panic disorder in adolescence. Journal of Clinical Child Adolescent Psychology. 2010;39(5):638-49.

29. Calamari JE, Hale LR, Heffelfinger SK, Janeck AS, Lau JJ, Weerts MA, et al. Relations between anxiety sensitivity and panic symptoms in nonreferred children and adolescents. J Behav Ther Exp Psychiatry. 2001;32(3):117-36.

30. Lau JJ, Calamari JE, Waraczynski M. Panic attack symptomatology and anxiety sensitivity in adolescents. J Anxiety Disord. 1996;10(5):355-64.

31. Leen-Feldner EW, Feldner MT, Bernstein A, McCormick JT, Zvolensky MJ. Anxiety sensitivity and anxious responding to bodily sensations: A test among adolescents using a voluntary hyperventilation challenge. Cognitive Therapy Research. 2005;29(5):593-609.

32. Borkovec TD, Nau SD. Credibility of analogue therapy rationales. J Behav Ther Exp Psychiatry. 1972;3(4):257-60.

33. American Psychiatric Association. Diagnostic and Statistical Manual of Mental Disorders. 5th ed. Arlington: American Psychiatric Association; 2013.

34. Arain M, Campbell M, Cooper CL, Lancaster GA. What is a pilot or feasibility study? A review of current practice and editorial policy. BMC Med Res Methodol. 2010;10(1):67.

35. Sim J, Lewis M. The size of a pilot study for a clinical trial should be calculated in relation to considerations of precision and efficiency. J Clin Epidemiol. 2012;65(3):301-8.

36. Julious SA. Sample size of 12 per group rule of thumb for a pilot study. Pharmaceutical Statistics: The Journal of Applied Statistics in the Pharmaceutical Industry. 2005;4(4):287-91.

37. Lancaster GA, Dodd S, Williamson PR. Design and analysis of pilot studies: recommendations for good practice. Journal of evaluation in clinical practice. 2004;10(2):307-12.

38. Malterud K, Siersma VD, Guassora AD. Sample size in qualitative interview studies: guided by information power. Qual Health Res. 2016;26(13):1753-60. 
39. Braun V, Clarke V. To saturate or not to saturate? Questioning data saturation as a useful concept for thematic analysis and sample-size rationales. Qualitative Research in Sport, Exercise and Health. 2019:1-16.

40. Chan A-W, Tetzlaff JM, Altman DG, Laupacis A, Gøtzsche PC, Krleža-Jerić K, et al. SPIRIT 2013 statement: defining standard protocol items for clinical trials. Ann Intern Med. 2013;158(3):200-7.

41. Law D, Wolpert M. Guide to Using Outcomes and Feedback Tools with Children, Young People and Families (Formally known as COOP (Children and Young Peoples' Improving Access to Psychological Therapies Outcomes Oriented Practice) version 2 of the above updated December 2013). CORC Ltd; 2014.

42. Clark DM, Salkovskis PM, Hackmann A, Middleton H, Anastasiades P, Gelder MG. A comparison of cognitive therapy, applied relaxation and imipramine in the treatment of panic disorder. The British Journal of Psychiatry. 1994;164(6):759-69.

43. Clark DM, Salkovskis PM. Panic Disorder. 2009.

44. Silverman WK, Albano AM. The anxiety disorders interview schedule for DSM-IV - child and parent versions. San Antonio.: Psychological Corporation; 1996.

45. Kaufman J, Birmaher B, Brent D, Rao U, Flynn C, Moreci P, et al. Schedule for affective disorders and schizophrenia for school-age children-present and lifetime version (K-SADS-PL): initial reliability and validity data. Journal of the American Academy of Child Adolescent Psychiatry. 1997;36(7):980-8.

46. Elkins RM, Gallo KP, Pincus DB, Comer JS. Moderators of intensive cognitive behavioral therapy for adolescent panic disorder: the roles of fear and avoidance. Child and Adolescent Mental Health. 2015:n/a-n/a.

47. Chorpita BF, Yim L, Moffitt CE, Umemoto LA, Francis SE. Assessment of symptoms of DSM-IV anxiety and depression in children: a revised child anxiety and depression scale. Behav Res Ther. 2000;38(8):835-55.

48. Chorpita BF, Moffitt CE, Gray J. Psychometric properties of the Revised Child Anxiety and Depression Scale in a clinical sample. Behav Res Ther. 2005;43(3):309-22.

49. Langley AK, Bergman RL, McCracken J, Piacentini JC. Impairment in Childhood Anxiety Disorders: Preliminary Examination of the Child Anxiety Impact Scale-Parent Version. J Child Adolesc Psychopharmacol. 2004;14(1):105-14.

50. Langley AK, Falk A, Peris T, Wiley JF, Kendall PC, Ginsburg G, et al. The child anxiety impact scale: examining parent-and child-reported impairment in child anxiety disorders. Journal of Clinical Child Adolescent Psychology. 2014;43(4):579-91.

51. Guy W. Clinical global impression scale. The ECDEU Assessment Manual for PsychopharmacologyRevised Volume. DHEW Publ No ADM. 1976;76(338):218-22.

52. Chambless DL, Caputo GC, Bright P, Gallagher R. Assessment of fear in agoraphobics: The body sensations questionnaire and the agoraphobic cognitions questionnaire. J Consult Clin Psychol. 1984;52(6):1090. 
53. Chambless DL, Caputo GC, Jasin SE, Gracely EJ, Williams C. The mobility inventory for agoraphobia. Behav Res Ther. 1985;23(1):35-44.

54. Miller SD, Duncan BL, Brown J, Sparks JA, Claud DA. The outcome rating scale: A preliminary study of the reliability, validity, and feasibility of a brief visual analog measure. Journal of brief Therapy. 2003;2(2):91-100.

55. Bringhurst DL, Watson CW, Miller SD, Duncan BL. The Reliability and Validity of the Outcome Rating Scale: A Replication Study of a Brief Clinical Measure. Journal of Brief Therapy. 2006;5(1):23-30.

56. Miller SD, Duncan BL, Johnson LD. The session rating scale 3.0. Chicago: Authors; 2000.

57. Duncan BL, Miller SD, Sparks JA, Claud DA, Reynolds LR, Brown J, et al. The Session Rating Scale: Preliminary psychometric properties of a "working" alliance measure. Journal of brief Therapy. 2003;3(1):3-12.

58. Campbell A, Hemsley S. Outcome Rating Scale and Session Rating Scale in psychological practice: Clinical utility of ultra-brief measures. Clinical Psychologist. 2009;13(1):1-9.

59. Law D, Jacob J. Goals and goal based outcomes (GBOs) London: CAMHS Press; 2015 [Available from: https://www.corc.uk.net/media/1219/goalsandgbos-thirdedition.pdf.

60. Astride-Stirling J. Development of Methods to Capture User Views of Child and Adolescent Mental Health Services for Clinical Governance Reviews. London: Commission for Health Improvement: Project Evaluation Report;; 2002.

61. Brown A, Ford T, Deighton J, Wolpert M. Satisfaction in child and adolescent mental health services: Translating users' feedback into measurement. Administration Policy in Mental Health Mental Health Services Research. 2014;41(4):434-46.

62. EuroQuol. European Quality of Life-5 Dimension 5-level: EQ-5D-5L. http://wwweuroquolorg/eq-5dproducts/eq-5d-5lhtml. 2015.

63. Dolan P, Gudex C, Kind P, Williams A. A social tariff for EuroQol: results from a UK general population survey. Centre for Health Economics University of York, UK; 1995.

64. Wille N, Badia X, Bonsel G, Burström K, Cavrini G, Devlin N, et al. Development of the EQ-5D-Y: a childfriendly version of the EQ-5D. Qual Life Res. 2010;19(6):875-86.

65. Ravens-Sieberer U, Wille N, Badia X, Bonsel G, Burström K, Cavrini G, et al. Feasibility, reliability, and validity of the EQ-5D-Y: results from a multinational study. Qual Life Res. 2010;19(6):887-97.

66. Stevens K. Valuation of the Child Health Utility 9D Index. Pharmacoeconomics. 2012;30(8):729-47.

67. Ratcliffe J, Stevens K, Flynn T, Brazier J, Sawyer M. An assessment of the construct validity of the CHU9D in the Australian adolescent general population. Quality of life research. 2012;21(4):717-25.

68. Stevens K, Ratcliffe J. Measuring and valuing health benefits for economic evaluation in adolescence: an assessment of the practicality and validity of the Child Health Utility $9 \mathrm{D}$ in the Australian adolescent population. Value in Health. 2012;15(8):1092-9.

69. Boyatzis RE. Transforming qualitative information: Thematic analysis and code development: sage; 1998. 
70. Braun V, Clarke V. Using thematic analysis in psychology. Qualitative Research in Psychology. 2006;3(2):77-101.

71. Braun V, Clarke V. What can "thematic analysis" offer health and wellbeing researchers? International Journal of Qualitative Studies of Health Well-being. 2014;9.

72. McManus F, Peerbhoy D, Larkin M, Clark DM. Learning to change a way of being: an interpretative phenomenological perspective on cognitive therapy for social phobia. J Anxiety Disord. 2010;24(6):581-9.

73. Beattie A, Shaw A, Kaur S, Kessler D. Primary-care patients' expectations and experiences of online cognitive behavioural therapy for depression: a qualitative study. Health Expect. 2009;12(1):45-59.

74. Yardley L. Dilemmas in qualitative health research. Psychology health. 2000;15(2):215-28.

\section{Figures}




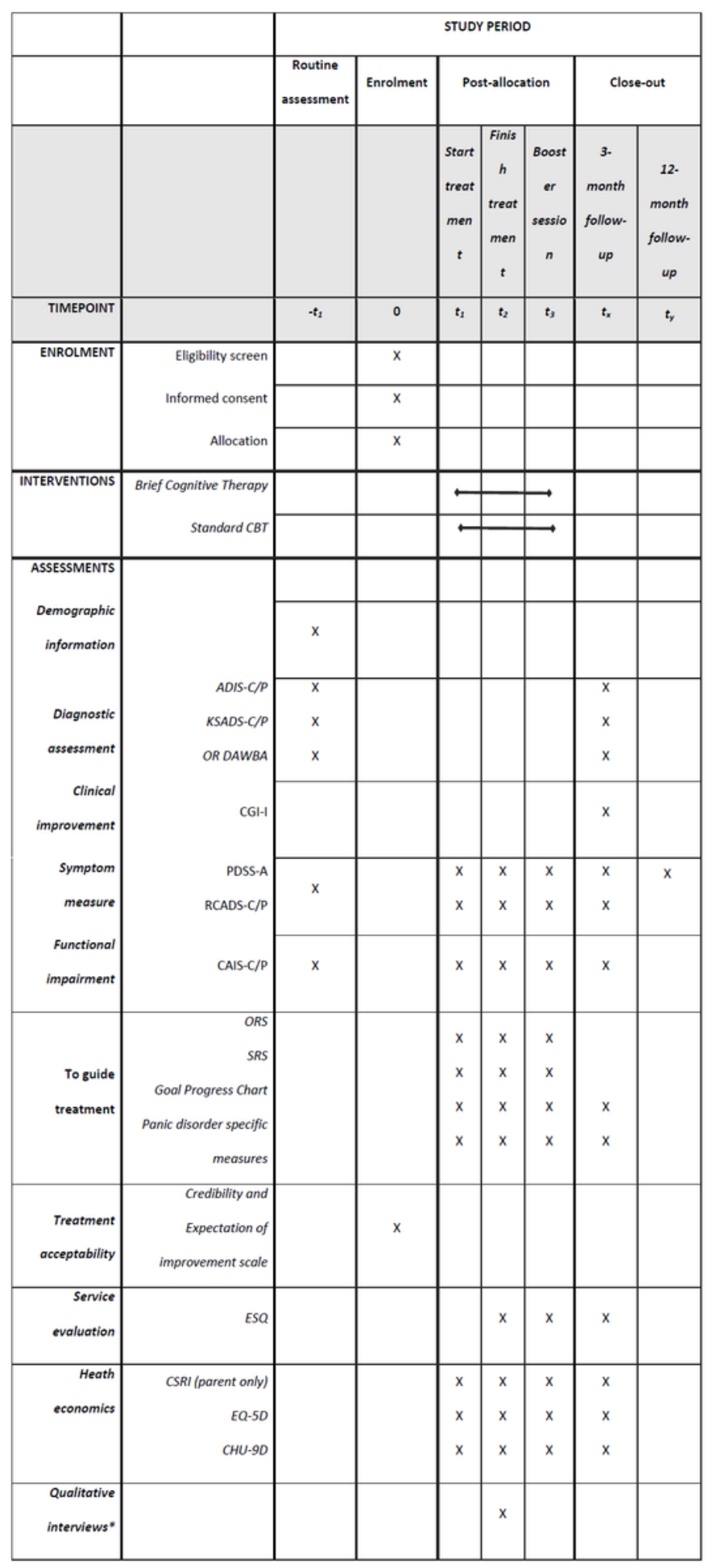

\section{Figure 1}

SPIRIT schedule of enrolment, interventions and assessments Note: * Qualitative interviews with a subsample of participants; ADIS-C/P = Anxiety Disorder Interview Schedule for DSM-IV child and parent version (anxiety section and common comorbid disorders); Panic disorder specific measures: ACQadapted = Agoraphobia Cognitions Questionnaire (adapted), BSQ-adapted = Body Sensations Questionnaire (adapted), SBQ-adapted = Safety Behaviour Questionnaire (adapted), MI-adapted = 
Mobility Inventory (adapted); CAIS-C/P = Child Anxiety Impact Scale - child and parent version; CGI-I = Clinical Global Impression- Improvement; CHU-9D = Child Health Utility (Paediatric Quality of Life); CSRI = Client Services Receipt Inventory; EQ-5D-Y = EuroQol (Quality of Life); ESQ = Experience of Service Questionnaire; KSADS-C/P = Kiddie Schedule for Affective Disorders and Schizophrenia - child and parent version (depression screen and supplement (including persistent depressive disorder), mania screen (supplement only if screening questions are endorsed)); ORS = Outcome Rating Scale; PDSS-A = Panic Disorder Severity Scale for Adolescents; RCADS-C/P = Revised Child Anxiety and Depression Scalechild and parent versions; SRS = Session Rating Scale. 


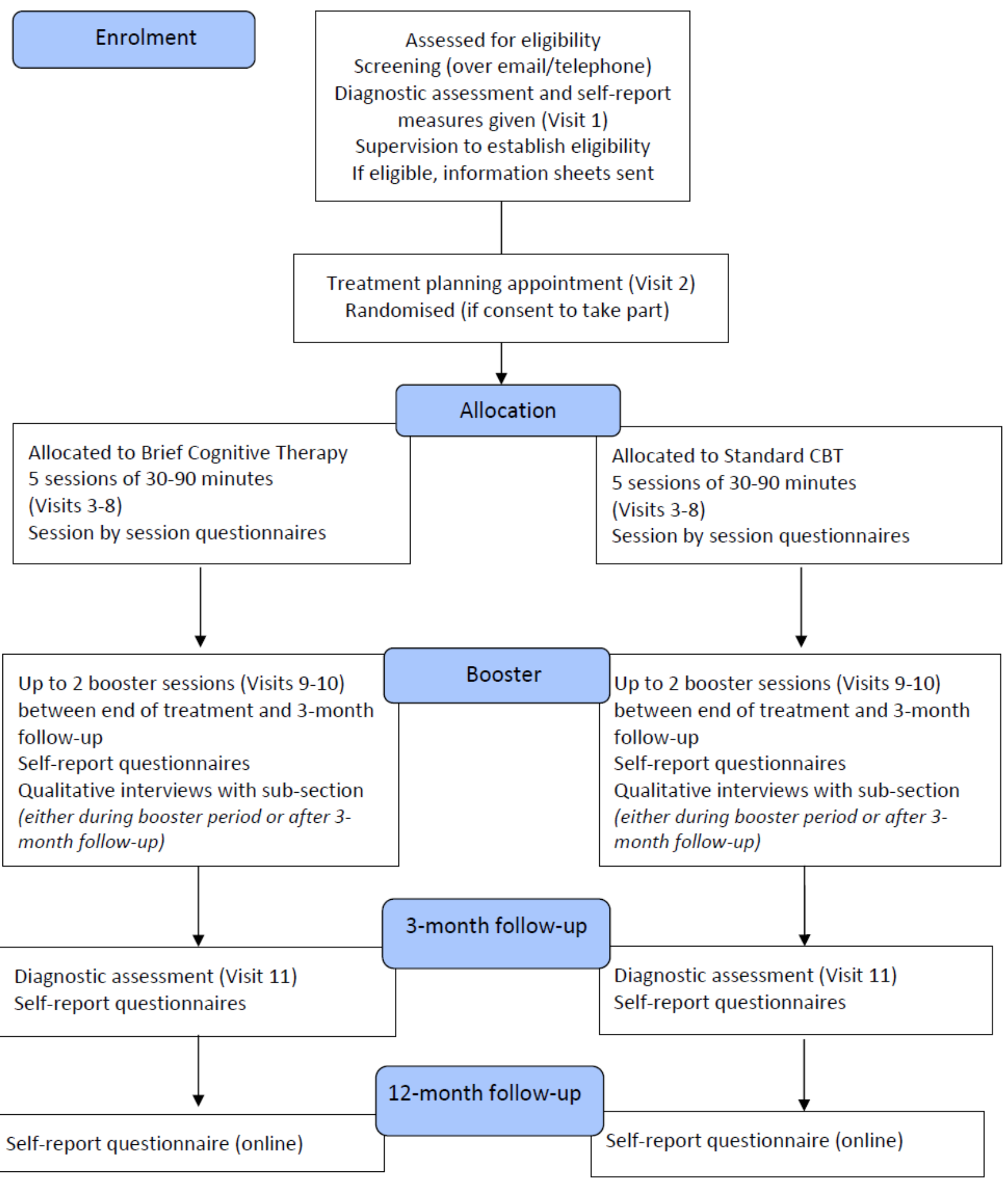

\section{Figure 2}

Overview of study procedure

\section{Supplementary Files}

This is a list of supplementary files associated with this preprint. Click to download. 
- PANDAESM310821.docx 